\title{
Mayak, Tscheljabinsk, Tschernobyl und die Folgen
}

Jürg Ulrich

Korrespondenz:

Prof. Dr. med. Jürg Ulrich Grellingerstrasse 65

CH-4052 Basel

juerg.ulrich@unibas.ch
Es gibt ärztliche Anliegen, die alle Menschen besonders stark beschäftigen. Dazu gehört, die Radioaktivität, der wir ausgesetzt sind, minimal zu halten. Atombombenversuche - auch wenn sie lange zurückliegen - und Unfälle in Nuklearkraftwerken erhöhen sie drastisch. Kenntnis über die dadurch erzeugten Gesundheitsschäden zu erwerben ist nicht nur ein Bedürfnis von Ärzten, sondern auch von historisch und politisch Interessierten; denn die damit verbundenen Probleme stehen in einem tragischen welthistorischen Zusammenhang. Ihn zu verstehen ist notwendig, wenn man weitere Schäden vermeiden will. Deshalb haben Basler Ärzte, Doppelmitglieder der Vereinigung «Ärzte für die Vermeidung eines Atomkriegs» (IPPNW) und des «Freundes- und Förderkreises Osteuropa» (FFKOe), eine Arbeitsgruppe gegründet, die Ausmass, Konsequenz, Geschichte und geographische Verteilung der Verseuchung in der ehemaligem Sowjetunion studiert. Hier sind besonders weite Gebiete verstrahlt - nicht nur durch Atombombenversuche und «Chernobyl», sondern auch infolge des schweren atomaren Unfalls in Mayak (Ural). Die Arbeitsgruppe führte bisher zwei öffentliche Anlässe zum Thema «Ungang mit Kernwaffen und Kernenergie in den Ländern der Ex-Sowjetunion» durch.

Der erste Anlass fand am 27. September 2007 im Hörsaal des Instituts für Pathologie am Universitätsspital Basel statt. Nach Vorträgen von Prof. A. Nidecker, Dr. C. Knüsli und dem Schreibenden folgte eine allgemeine Diskussion. Referiert wurde über Geschichte der Nuklearexplosionen und Reaktorzwischenfälle in der Sowjetunion, über die medizinischen Folgen der Katastrophe von Tschernobyl 1986 und die Möglichkeiten, Kindern aus den verseuchten Gebieten zu helfen. Die Zuhörer wurden damit auf den bereits geplanten zweiten Anlass vom 26. April 2008 vorbereitet. Dr. Knüsli referierte über das vermehrte Vorkommen verschiedener Tumore und anderer Krankheiten in den von «Tschernobyl» betroffenen Gebieten und orientierte über eine Abmachung zwischen WHO und IAEO. Infolge dieses Vertrags übt letztere de facto eine
Zensur über die Publikationen der WHO aus. Prof. Nidecker zeigte, wie ärztliches Handeln den Zustand von Kindern mit Strahlenschäden bessern kann, wenn man ihnen unverstrahlte Nahrung und Pektin verabreicht.

Am 26. April 2008 wurde an einem ganztägigen Anlass im Kollegiengebäude der Universität Basel der Opfer der Katastrophe von Tschernobyl gedacht. Russische und weissrussische Ärzte, Biologen und Historiker waren anwesend und referierten. Prof. Dr. Carsten Goehrke, Zürich, emeritierter Ordinarius für osteuropäische Geschichte, leitete die Vormittagssitzung. Im Anschluss sprach Dr. Novoselov, Wissenschaftshistoriker aus Tscheljabinsk (Russland), über die Entwicklung der Nuklearindustrie in der UdSSR, besonders über ihre Folgen für Umwelt und Gesellschaft. Die UdSSR rüstete ab 1943 nuklear auf, gewann uranhaltige Erze und reicherte dieses Element in speziellen Anlagen an. In Mayak arbeiteten zeitweise 100000 Mann - besonders Kriegsgefangene und Soldaten. 1948 wurde es hier möglich, 100 Gramm Plutonium pro Tag zu produzieren. Bekanntlich wurde schliesslich im August 1949 in der Nähe von Semipalatinsk (Kasachstan) die erste sowjetische Atombombe gezündet. Dieser Explosion folgten weitere - u. a. in Novaja Zemlja (arktischer Ozean). Die Organisation dieser Industrie, eine Art Staat im Staat, schenkte damals den Umweltproblemen wenig Beachtung.

Dr. Svetlana Krasnoslobodseva schilderte die radioaktive Kontamination in sowjetischen nuklearen Einrichtungen und ihrer Umgebung. Sie verglich den Gesundheitszustand der Bevölkerung mit demjenigen der Mitarbeiter der Anlagen. Letztere waren betreut und wurden in Bezug auf Strahlenexposition geschont. Der Millionenstadt Tscheljabinsk und ihrer Umgebung wurde solche Schonung erst nach dem Unfall von 1957 zugestanden. Interessante Einzelheiten erzählten die beiden Referenten aus Tscheljabinsk in der Diskussion und im privaten Gespräch im Anschluss: Die Explosion erfolgte in einem Behälter, in dem radioaktiver Abfall deponiert war. Das Wasser desselben wurde in Folge der inten- 


\section{Abbildung 1}

22. Tschernobyl-Gedenktag (26. April 2008) vor dem Gästehaus des Universitätsspitals Basel: Von links nach rechts: Alexej Gusev (Moskau); Jürg Ulrich (Basel); Sandrine Mayoraz (Basel); Rose Goncharova (Minsk); Svetlana Krasnoslobodseva (Tscheljabinsk); Alexej Yablokov (Moskau); Vassilij Nesterenko (Minsk).

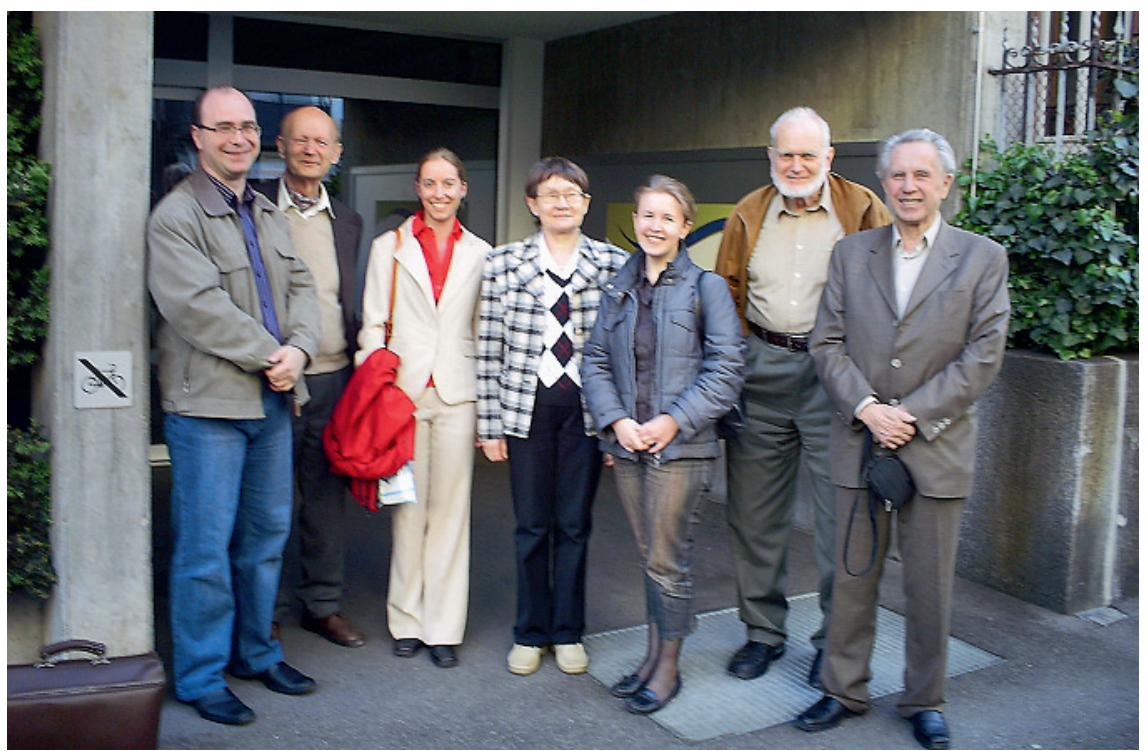

siven Bestrahlung dermassen erhitzt, dass der Dampfdruck zur Explosion führte. Ein mehrere Meter dicker Betondeckel flog in die Luft. Dem Behälter entstieg ein Rauch- und Staubpilz, ähnlich denjenigen von Atombombenexplosionen. Das Gerücht kam auf, die Amerikaner hätten eine Atombombe abgeworfen. Aus dem «Pilz» fiel in Folge hochgradig radioaktive Asche nieder.

Hier fragt sich, ob die Sicherheit nuklearer Installationen tatsächlich nur ein technisches Problem sei. Psychologische Spannungen sind in der stark hierarchisch organisierten Belegschaft kaum zu vermeiden, und ein falsches Sicherheitsgefühl kann die Ursache für folgenreiche Fehlmanipulationen werden.

Am Nachmittag lenkten die Referenten die Aufmerksamkeit auf die medizinisch-biologischen Folgen der Katastrophe in Tschernobyl (Weissrussland/Ukraine). Dr. Rose Goncharova vom Institut für Genetik und Zytologie der weissrussischen Akademie der Wissenschaften in Minsk wies darauf hin, dass nicht nur der Schilddrüsenkrebs bei Kindern und Erwachsenen in den verseuchten Gebieten zunimmt, sondern auch andere Krebsarten. Besonders alarmierend sind Chromosomenanomalien bei gezüchteten Waldmäusen, Nachkommen verstrahlter Wildtiere.
Der seither verstorbene Professor V. B. Nesterenko vom Institut BELRAD für Strahlensicherheit in Minsk beschrieb den Anblick des zerstörten Reaktors von Tschernobyl vom Helikopter aus. Als einer der ersten hatte er diesen nach dem Unfall gesehen. Sodann vermittelte er einen Eindruck der radioaktiven Verseuchung in Weissrussland und beschrieb von ihm konstruierte Apparate zur Messung der Radioaktivität im Inneren des Körpers von Exponierten. Darauf gestützt beraten Mitarbeiter des Instituts die exponierte Bevölkerung und führten eine eigentliche radioökologische Erziehung durch. Verminderungen der inneren Bestrahlung nach Behandlungen mit dem Pektinpräparat «Vitapect» sind leider oft von kurzer Dauer, denn die Exponierten, meist Kinder, sind oft schwer davon abzuhalten, Pilze und Beeren aus den verstrahlten Wäldern zu geniessen.

Schliesslich fasste Prof. A. V. Yablokov, Biologe in Moskau und Berater der Russischen Akademie der Wissenschaften, zusammen: 1. Der Zusammenbruch der Sowjetunion hing sehr eng mit der Katastrophe von Tschernobyl zusammen; 2. Nuklearingenieure sind oft allzu sorglos und neigen mit anderen Experten für Nuklearenergie dazu, deren Gefahren zu unterschätzen; 3. Terrorismus und Ignoranz sind in nuklearen Installationen besonders gefährlich; 4. die Existenz der rund 450 Reaktoren auf unserem Planeten ist wegen dieser Gefahren eine Bedrohung der ganzen Menschheit; 5. die Kosten der Sicherheitsmassnahmen allein sind enorm (etwa 500 Milliarden Dollar); 6. die Sterblichkeit durch vermehrte Radioaktivität in kontaminierten Gebieten liegt um $4 \%$ über der allgemeinen Durchschnittssterblichkeit; 7. Die Nuklearenergie bringt mit sich, dass die Menschenrechte enorm eingeschränkt werden - sie stellt nicht nur das Recht auf gesunde Umgebung infrage, sondern führt auch zu einer vermehrten Überwachung der Bevölkerung (wegen der Gefahr von Terrorakten).

Die Arbeitsgruppe zur Radioaktivität in der Ex-Sowjetunion ist zurzeit im Begriff, ihre Kenntnisse zu vertiefen und zu erweitern. In diesem Rahmen werden gemeinsam wissenschaftliche Tagungen besucht, und man orientiert sich gegenseitig über neue Entwicklungen - und dies vorwiegend in kleinen Gruppen. Die Mitwirkung weiterer Interessierter ist durchaus erwünscht. Neue öffentliche Anlässe sind vorgesehen. 